\title{
18 Jahre Allergo Journal - Ringen für eine bessere Allergologie
}

D ies ist eine besondere Ausgabe von Allergo Journal, denn mit diesem Heft verabschieden sich die beiden langjährigen Herausgeber Gerhard Schultze-Werninghaus und Bernhard Przybilla - Grund genug, zusammen mit dem Ehrenherausgeber Johannes Ring auf 18 Jahre Allergo Journal zurückblicken.

Ende der 80er Jahre war es vielen jungen Allergologen und Allergieforschern in Deutschland ein Bedürfnis, neben der bekannten „Allergologie“ eine neue, schwungvolle und attraktive Zeitschrift für den deutschsprachigen Leserkreis zu schaffen, die einerseits gute Wissenschaft, aber auch aktuelle Information, kontroverse Diskussionen, Interviews und Standespolitik anbieten könnte. Sie sollte attraktiv aufgemacht sein und gerne gelesen werden.

Lange wurde das Sprachenproblem diskutiert: Gleich ganz Englisch? Oder Deutsch mit englischen Kurzfassungen? Schließlich entschied man sich für Letzteres, nahm aber auch englische Originalarbeiten und Übersichten an, die jedoch im Laufe der Zeit immer seltener wurden. Es stellte sich heraus, dass für die deutsche Leserschaft auch eine deutschsprachige Zeitschrift gebraucht wird, um in die Breite zu wirken.

Es war unter der Präsidentschaft von Wolfgang Schmutzler der damals noch „Deutsche Gesellschaft für Allergie und Immunitätsforschung" (DGAI) genannten Gesellschaft, als sich Gerhard Schultze-Werninghaus und Johannes Ring zusammen mit Kollegen des späteren Herausgeberkollegiums und dem auf Wolfgang Schmutzler folgenden DGAI-Präsidenten Gert Kunkel zu intensiven Verhandlungen mit Jochen Aumiller trafen - damals Chefredakteur der Münchner Medizinischen Wochenschrift

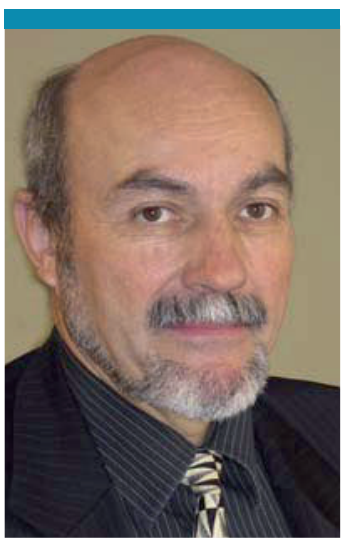

Prof. Dr. Dr. Johannes Ring, Klinik und Poliklinik für Dermatologie und Allergologie am Biederstein, Technische Universität München

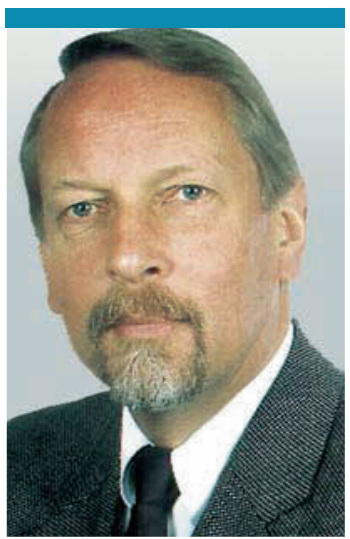

Prof. Dr. Gerhard SchultzeWerninghaus, Abteilung für Pneumologie, Allergologie und Schlafmedizin, Medizinische Klink III, BG-liches Universitätsklinikum Bergmannsheil, Bochum

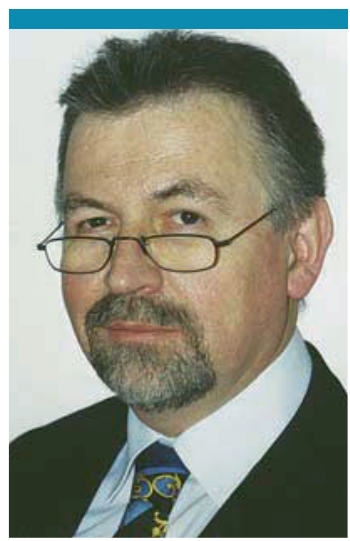

Prof. Dr. Bernhard Przybilla, Klinik und Poliklinik für Dermatologie und Allergologie, Klinikum der LudwigMaximilians-Universität, München
-, um das Konzept der neuen Zeitschrift zu entwickeln. Schon die Wahl des $\mathrm{Na}$ mens sollte eine spätere Öffnung in den internationalen Raum ermöglichen: Allergo Journal liest sich auf Deutsch, Französisch und Englisch gleich gut.

Die Zeitschrift sollte ansprechend sein in der Machart. Das jeweilige Titelblatt verlangte nach Signalcharakter: Man wollte weder langweilige Inhaltsverzeichnisse noch bunte Karikaturen oder Werbebilder - und entschied sich zur schwarzweißen Wiedergabe von meist rasterelektronenmikroskopischen Bildern, die über die Jahrzehnte hin meisterlich von Prof. Dr. Heidrun Behrendt, später in $\mathrm{Zu}$ sammenarbeit mit Dr. Ingrid Weichenmeier in exzellenter Qualität geliefert wurden. Man könnte daraus einen „Ultrastruktur-Atlas der Allergologie" machen. Die Bilder verbinden wissenschaftliches Denken und Ästhetik in vornehmer Weise und sind zum Markenzeichen des Allergo Journal geworden.

Auf dem Kongress in Potsdam 1993 gelang es, die Mitglieder der DGAI davon zu überzeugen, Allergo Journal als offizielles Organ der Gesellschaft zu akzeptieren und zusammen mit dem Mitgliedsbeitrag fest zu abonnieren. In kurzer Zeit erreichte Allergo Journal eine Auflage von 7.000 bis 8.000 Exemplaren im deutschen Sprachraum.

Die neue Zeitschrift war nicht als gegen die bestehende „Allergologie“ gerichtet gedacht. Und es stellte sich tatsächlich bald heraus, dass Bedarf für zwei allergologische Fachzeitschriften in Deutschland bestand. Schon kurz darauf, im Jahr 1994, gelang es, auch den Ärzteverband Deutscher Allergologen (ÄDA) mit ins Boot zu bekommen, so dass Allergo Journal heute das offizielle Organ beider großer Fachgesellschaften darstellt.

Es gibt noch einen weiteren Grund, warum dieses Heft 8/09 von Allergo Jour- 
nal, das Sie in Händen halten, eine besondere Ausgabe ist: Es ist Herrn Professor Dr. Erich Fuchs gewidmet, dem langjährigen Präsidenten der DGAI (1978-1987), der auch Ehrenherausgeber der Zeitschrift war. Er ist am 5. Oktober 2008 im 87. Lebensjahr verstorben. Er hat die deutsche Allergologie durch sein Wissen, seine kritische wissenschaftliche Grundhaltung sowie seine integrative Kraft in der Brückenbildung von der praktischen Allergologie hin zur modernen Allergieforschung entscheidend geprägt.

Ihm gewidmet sind vier Arbeiten aus verschiedenen Teilen des großen Gebietes der Allergologie in diesem Heft: Die Beziehungen zwischen Infektion, Umweltbelastung und Allergie werden am Beispiel von viralen Atemweginfektionen bei Asthma bronchiale behandelt (Rohde, S. 596), ebenso die Fortschritte der modernen molekularen Genetik im Hinblick auf die Erkennung pathophysiologisch relevanter Mutationen des epidermalen Proteins Filaggrin bei atopischem Ekzem (Weidinger et al., S. 600). Stellvertretend für neue Therapeutika wird die Therapie mit monoklonalen Antikörpern gegen Immunglobulin E - Omalizumab - bei schwerem Asthma vorgestellt (Korn et al., S. 610). Schließlich erfährt die einzige kausale Therapie neben der Allergenvermeidung - die allergenspezifische Immuntherapie - eine Würdigung (Ruëff et al., S. 618).

Über die Jahrzehnte hinweg haben meist die Chefherausgeber des Allergo Journal den Leitartikel „Zur Sache“ gemeinsam geschrieben, wobei der links Unterzeichnete schriftstellerisch in Vorlage ging. Zu speziellen Heften kamen auch andere Autoren im Editorial zum Zug. Man soll sich nicht selbst loben, aber den Autoren von „Zur Sache“ schlugen in den vergangenen 18 Jahren neben manch berechtigter Kritik doch viel mehr Lob und
Zustimmung entgegen. Viele Kollegen sagten uns: „Das Allergo Journal mache ich gerne auf, das spricht mich an, auch wenn ich nicht alles lese."

Allergo Journal ist auch Konfrontationen nicht ausgewichen: Wir kämpften mit dem Verlag, um Anzeigen für unwissenschaftliche „komplementäre" Therapieverfahren zu verhindern. Wir kämpften gegen schlechte Wissenschaft und konnten mitteilen, dass Raubmilben keinen Kot produzieren, so dass auch eine Allergenverwandtschaft zwischen Hausstaubmilben- und Raubmilbenkot eher im Bereich der virtuellen Allergologie anzusiedeln ist.

Herausgeber einer solchen Zeitschrift zu sein, macht viel Arbeit, aber noch mehr Spaß. Wir freuen uns, dass es gelungen ist, als Vertreter der beiden Verbände hervorragende Nachfolger im Amt der Herausgeber zu finden, die sich im neuen Jahr 2010 vorstellen werden. Ein bisschen Spannung darf sein!

Ein Wermutstropfen war das vergebliche Bemühen um die Zuteilung eines „Impact Factor", d.h. die Aufnahme in Index Medicus und Medline. Wir haben es alle drei Jahre versucht, haben stets die formalen Kriterien - wie z. B. „peer-reviewed" Originalia und Übersichten sowie englische Kurzfassungen und „key words" - erfüllt und wurden doch nie akzeptiert. Mittlerweile glauben wir zu wissen, dass dies nicht am Inhalt liegt. Die Personen, die in Washington, D.C., darüber entscheiden, glauben wohl, dass eine wissenschaftliche Zeitschrift langweilig aussehen muss, und werfen ein Heft, das auch aktuelle Informationen und ansprechendes Bildmaterial enthält, weg. So jedenfalls vermutet einer der Unterzeichneten. Dennoch sollten wir nicht müde werden. Wir sind sicher, dass Allergo Journal einen echten „Impakt“ auf die deutsche Allergologie hat, unabhängig vom rechnerisch zugeteilten Faktor.

In den 18 Jahren Allergo Journal haben wir um eine bessere Allergologie in Deutschland gerungen, um eine bessere Versorgung allergiekranker Menschen in unserem Lande. Damit meinten wir eine Aufwertung der Zusatzbezeichnung „Allergologie“ nicht nur in der inhaltlichen Qualität der Weiterbildung, sondern vor allem in der praktischen Bedeutung im Hinblick auf die Patientenversorgung und Leistungsabrechnung. Das ist uns und den vielen Mitstreitern nicht gelungen. Allergologen sind in Deutschland eine Minderheit, die von den zahlenmäßig viel größeren Fachgesellschaften und Hausarztverbänden einfach überstimmt wird. Der damalige Gesundheitsminister Horst Seehofer sagte uns einmal: „Ich verstehe Ihr Problem, Sie brauchen einen Minderheitenschutz wie die Indianer!" Unsere Verbände unter neuer Führung werden dieses Bemühen fortführen und hoffentlich eines Tages erfolgreich sein. Es gibt Überlegungen, die Einführung eines Facharztes für Allergologie nach dem Schweizer Modell zu fordern, der dann gleichzeitig mit einem organbezogenen Facharzttitel geführt werden darf. Bis so etwas erreicht wird, wird aber noch viel Wasser den Rhein, die Donau und die Elbe hinabfließen; aber wer soll so etwas fordern, wenn nicht wir? Die organbezogenen Fachgebiete würden darunter nicht leiden.

Nun wünschen wir unseren Nachfolgern in der Herausgeberschaft eine glückliche Hand, viel Geduld und Kraft, aber besonders Freude an der kreativen Aufgabe. Ihnen, der Leserschaft, ein besinnliches Weihnachtsfest und ein erfolgreiches und friedliches Jahr 2010!

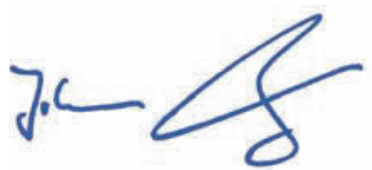

Prof. Dr. Dr. Johannes Ring

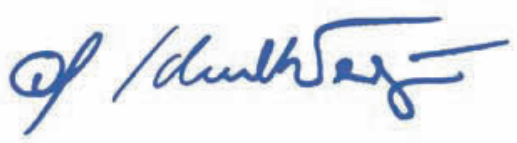

Prof. Dr. Gerhard Schultze-Werninghaus

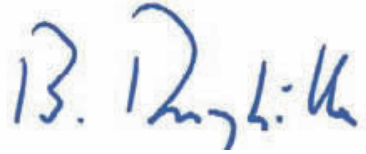

Prof. Dr. Bernhard Przybilla 